\title{
Exilé(e)s et migrant(e)s transatlantiques: histoires entremêlées, historiographies parallèles
}

Sylvie Aprile* Université Paris Nanterre, Laboratoire ISP

Nanterre - França

L'historienne du XIXe siècle ne peut pas être insensible au présent et c'est avec ce présent en tête, avec les images actuelles de la question des réfugiés qu'il travaille aujourd'hui sur l'exil politique d'hier. Cette actualité inscrit peu à peu cette recherche dans un champ plus vaste : celui des circulations et des mobilités. Vue de France, la question des exilés a pris une grande ampleur depuis une décennie et l'angle choisi depuis les premiers travaux a orienté l'analyse vers une histoire sociale du politique. Si l'arrivée des exilés européens en France, vu comme 
une terre d'accueil a été un terrain très fécond, j’ai privilégié un autre regard, consacré au départ des Français au cours du XIXe siècle vers des destinations proches ou lointaines et notamment vers l'Amérique $^{1}$. Dans tous ces pays, les Français rencontraient d'autres exilés européens et d'autres cultures qui ont orienté ma démarche vers une réflexion plus transnationale et globale.

Evoquer les migrations à propos de la France conduit en effet généralement à s'interroger sur la réception, c'est-à-dire l'accueil ou le rejet par les Français des immigrés, à faire une histoire des politiques de l'état français à l'égard des étrangers, à répertorier les motivations et les appartenances communautaires de tous ceux qui sont venus s'installer en France pour des raisons économiques et politiques. On évoque moins souvent et à juste titre car ils sont bien entendu bien moins importants quantitativement les mouvements des Français et leur apport à une histoire mondiale des migrations volontaires ou contraintes. Ces migrations ont malgré tout existé et alimenté des imaginaires sociaux et politiques similaires à ceux des autres Européens ${ }^{2}$ Je souhaiterais ici revenir sur l'état de la question en reprenant des travaux déjà existants, menés notamment dans le cadre nord et sud américains et développer quelques pistes de réflexions historiographiques nées de mes travaux. Mon approche est en effet aussi différente car je n'ai pas suivi des groupes, pas analysé des lieux particuliers d'implantation mais suivi des trajectoires particulières d'hommes et de femmes qui partent ici outre-Atlantique tout à la fois par la contrainte mais aussi par choix ou après d'autres expériences d'exil. Cette analyse rejoint les travaux menés aujourd'hui dans le champ des études migratoires en terme de mobilité et de circulation et non plus d'intégration. Les questions du transit, de l'attente, du retour, en résumé de toutes les formes d'instabilité que vivent les migrants d'hier et d'aujourd'hui sont présentes dans

1 Delphine Diaz, Un asile pour tous les peuples? Exilés et réfugiés étrangers dans la France du premier XIX siècle, Paris, Armand Colin, 2014, collection « Recherches ».

2 Olivier Brégeard, «Une communauté fragile, les Français de New York au milieu du XIXème siècle», Annales de démographie historique, 2001, p. 43-53. 
mon enquête, les exilés politiques ne pensant par leur séjour hors de France comme définitif, subissant ou choisissant des lieux successifs de résidence.

C'est donc à une réflexion sur la lecture croisée des historiographies des migrations et de l'exil que je souhaite ici me livrer avant de suivre les trajectoires de trois exilés. Tout d'abord deux exilés qui quittent la France pour l'Amérique pour fuir l'arrivée au pouvoir de Napoléon III. En dépit d'une apparente similitude biographique (même point de départ : la France, même cause politique : la proscription sous le second Empire), les histoires de ces deux hommes se ressemblent peu car elles sont marquées par des sinuosités qui les différencient et accroissent la toile complexe des connexions possibles. J'y ajouterai le parcours d'une femme, car elle sont aussi plus invisibles encore : Herminie Cadolle, exilée après la Commune de Paris devenue une commerçante florissante à Buenos Aires et dont la trajectoire témoigne également d'une histoire à la fois singulière et représentative des parcours complexes de la migration contrainte.

\section{Deux historiographies parallèles se rencontrent-elles?}

L'histoire de l'exil et celle de la migration outre atlantique se sont longtemps ignorées. Si elles se complètent aujourd'hui, elles ne se confondent et ne se confrontent encore que rarement : elles appartiennent encore souvent à des registres et à des champs académiques distincts. Rares sont les auteurs, spécialistes de l'histoire des migrations qui traitent à parts égales des différentes sortes de migrations. Les travaux sont orientés plus volontiers vers les migrations de masse que vers celles, plus ponctuelles ou quantitativement plus faibles, des exilés politiques. C'est le cas notamment de l'ouvrage, au demeurant tout à fait remarquable, de Philippe Rygiel, intitulé Le temps des migrations blanches ${ }^{3}$. Même lorsqu'ils mettent en avant une réflexion transnationale, rares sont les

3 Philippe Rygiel, Le temps des migrations blanches : migrer en Occident (1840-1940), Paris, Publibook, 2010 (2007). 
travaux qui échappent à ce cloisonnement. Certes, la quantification donne raison à cette inclination du regard et de la recherche. Mais c'est aussi une volonté de distinction qui écarte l'étude du migrant de celle de l'exilé. Le premier a longtemps été analysé par sa seule motivation économique qui aurait pour conséquence un seul objectif, celui de s'installer durablement. Au contraire, la nostalgie de tout exilé le conduirait à toujours penser au pays de départ et à envisager son séjour même lointain comme provisoire. En résumé le premier regarderait toujours vers l'avenir, l'autre vers le passé. Ces visions interdisent de penser des mouvements circulaires, des étapes, des formes de nomadismes concertées ou contraintes. Des travaux récents ont rapproché la situation du migrant et du réfugié en refusant ces causalités uniques. Du côté de l'histoire des migrations on peut citer l'ouvrage dirigé par Donna Gabaccia, Italy's Many Diasporas à partir d'un pays de départ, l'Italie, étudie par exemple une pluralité de lieux d'accueil et de figures de la migration ${ }^{4}$. J'ai tenté dans mes recherches sur l'exil de montrer que le choix d'un pays d'accueil relève non d'une volonté politique mais souvent d'une réflexion socio-économique : langue commune, opportunité de trouver un emploi. Les contraintes imposées aux exilés sont d'ailleurs souvent plus économiques que politiques : la Belgique leur impose en 1852 des conditions de ressources pour s'installer, la Grande Bretagne favorise le départ des plus pauvres vers les Etats-Unis, de nombreux pays mettent en place des législations contraignantes pour les professions libérales notamment les médecins qui ne peuvent pas y exercer leur métier.. L'historien Klaus Bade a émis une hypothèse très féconde dans son analyse des départs massifs d'Allemands vers l'Amérique aux lendemains de la répression du Printemps des peuples aux débuts des années $1850^{5}$ Dans son ouvrage, L'Europe en mouvement. La migration de la fin du XVIIIe siècle à nos jours, il développe une hypothèse intéressante sur la double acception du terme, « émigration de

4 Donna Gabaccia, Italy's Many Diasporas : Elites, Exiles and Workers of the world, Washington, University of Washington Press, 2000.

5 Klaus Bade, L'Europe en mouvement. La migration de la fin du XVIIIe siècle à nos jours, Paris, Seuil, 2002. 
soupape », l'entendant à la fois dans un sens classique c'est-à-dire socio-économique mais également politique. Selon lui, l'émigration a été pour beaucoup de ceux qui l'ont pratiqué, une sorte de protestation sociale muette, un « vote avec les pieds », contre les conditions de vie mais aussi l'absence de liberté d'expression qu'ils subissaient dans leur pays, l'émigration permettant d'y échapper sans avoir à lutter pour sa transformation $»^{6}$. Il conclut : «Supposons que des dizaines de millions d'émigrés n'aient pas pu quitter l'Europe du XIXe et du début du XXe siècle. Supposons par ailleurs que tous les autres, ceux qui restèrent bien qu'ils aient eu la possibilité d'émigrer, n'aient pas connu au XIXe siècle cette vision du Nouveau Monde considéré comme un échappatoire et une alternative tout à fait praticable au combat pour une vie meilleure : alors, le pronostic formulé par Karl Marx et Friedrich Engels, dont on sait, rétrospectivement, et selon lequel l'échec des révolutions politiques de 1848-1849 en Europe serait suivi par la réussite d'une révolution sociale internationale, paraîtrait peut être au bout du compte moins illusoire qu'il ne le fut ${ }^{7} »$. Quitter son pays serait alors à la fois une nécessité économique et une forme de résistance. L'idée est séduisante et met au jour une réalité largement confirmée par notre présent où les réfugiés peuvent à la fois fuir un régime qui les opprime et chercher de meilleures conditions de vie. Néanmoins, l'historien comme l'observateur contemporain cherche toujours à distinguer les migrants et les exilés même s'ils partagent une même trajectoire, à isoler pour faire vite le social du politique. On veut toujours distinguer le vrai et le faux migrant, le vrai et le faux réfugié, et plus encore toujours le migrant et le réfugié. Les situations communes sont encore trop souvent considérées comme fortuites ou conditionnées par le télescopage d'un événement historique que serait un phénomène de départ (push) alors même qu'un contexte économique semble alors déterminant comme phénomène d'attraction (pull). C'est le cas notamment,

6 Klaus Bade, L'Europe en mouvement, La migration de la fin du XVIIIe siècle à nos jours, Paris, Seuil, 2002, p 210.

7 Ibidem, p 209-210. Albert O Hirschman, Exit, voice, loyalty. Défection et prise de parole, (1970), Bruxelles, Editions de l’Université de Bruxelles, 2011. 
entre 1849 et 1851, de la ruée vers l'or qui a lieu au même moment que des départs d'exilés français vers la Californie étudiés par Annick Foucrier, ou des créations des communautés utopistes analysées par Ronald Creagh ${ }^{8}$. Faut-il tenter de dissocier ceux qui partent faire fortune de ceux qui veulent changer la société ? La représentation du Nouveau Monde est tout à la fois celle d'un espace vierge, d'un eldorado et d'une terre promise démocratique. L'histoire migratoire enrichit donc celle de l'exil. J'en donnerai deux exemples. En premier lieu, centrée sur le travail et le monde économique, l'approche migratoire restitue un quotidien, là où le vécu au jour le jour de l'exil est souvent peu pris en compte. Second point : comme pour les migrants économiques, le départ des exilés est encadré par une chaîne migratoire que l'on néglige souvent. Pour les élites de l'exil, du moins, on sait que la recommandation par une lettre du géographe, naturaliste Alexandre de Humboldt a ainsi constitué un sésame, une porte d'entrée aussi déterminante que celle de la protection d'un homme politique fut il célèbre. C'est le cas par exemple pour Amédée Jacques, en partance vers l'Uruguay en 1849. Remarquons au passage, que cet appui oriente de nombreux exilés vers des emplois de cartographes, de géographes, de photographes comme Victor Frond dont nous parlerons plus tard. Ils se fondent alors dans une autre catégorie, celle des voyageurs, elle aussi trop souvent écartée de l'histoire des migrations et des exils. Le goût de l'aventure et du hasard cadre mal avec la contrainte et avec la nécessité de gagner sa vie. Pourtant l'exil peut comme la migration se transformer souvent en une circulation qui devient un véritable mode de vie. Elisée Reclus a ainsi parcouru l'Amérique du Nord et du Sud après 1851, et publié ensuite les récits de ces voyages et réflexions géographiques. Pendant la Révolution française, Talleyrand de Périgord évoquait

8 Annick Foucrier, «Les exilés républicains français de 1848 et 1851 en Californie », in Catherine Collomp et Mario Menendez, dir., Exilés et réfugiés politiques aux États-Unis, 1789-2000, Paris, Editions du CNRS, 2003, p. 51-63. « Gold Rush California Bound: New Evidences from French Passenger Lists », in D.O. Flynn, A. Giraldez, and J. Soberdo, eds., Studies in Pacific History: Economic, Social and Political History, Londres, Ashgate Publishing Co., 2002, p. 95-107. Ronald Creagh, Utopies américaines, Marseille, Agone, 2010. 
dans ses souvenirs le hasard qui nous échappe souvent et nous conduit à établir des parcours comme s'ils étaient déjà tracés à l'avance. Ayant quitter Falsmouth, il arrive en Amérique mais avoue avoir souhaité repartir vers d'autres horizons :

«Après quelques semaines de navigation, je fus un matin réveillé par le cri que je redoutais de: Terre! Terre! Le capitaine, l'équipage et les passagers, tous montraient la joie la plus impatiente en montant sur le pont, j’aperçus en même temps et le pilote qui venait pour nous faire remonter la Delaware, et un vaisseau qui quittaient les caps. Fe demandai au pilote qu'elle était la destination du bâtiment que je voyais. Il me dit qu'il faisait voile pour Calcutta. F'envoyai sur le champ une barque pour lui faire demander s'il voulait prendre un passager. La destination du bâtiment m'importait peu; le voyage devait être long, et ce que je voulais c'était ne pas quitter la mer. Le nombre de passagers se trouvant complet, il fallut me laisser conduire à Philadelphie "s.

Cette citation nous permet également d'élargir à nouveau le champs spatial en quittant un cadre national mais aussi transatlantique. L'histoire impériale surtout anglo-saxonne a depuis quelques années étendu le regard de l'histoire des migrations à l'histoire coloniale. Partir loin en exil tout comme migrer ne signifie pas seulement emprunter la grande « route » transatlantique, d'autres opportunités se présentent : l'Inde, l'Australie, l'Algérie, elle peuvent servir d'étapes et de transits. Migrants et exilés ont aussi participé à la colonisation, sont passés d'une migration économique de l'Europe vers l'Amérique à des territoires extra-européens porteurs des mêmes aspirations à la découverte et au changement de vie. Les circulations sont multiples aléatoires.

\section{Exilés ou migrants, migrants ou exilés ? Campdoras et Frond $^{10}$}

Pour donner plus de chair à ce propos, il convient de suivre des itinéraires particuliers. J'ai choisi ceux de deux hommes, que je quali-

9 Mémoires du Prince de Talleyrand, p 232.

10 Pour une analyse plus approfondie de la vie de Campdoras, voir René Merle, Mille-huit cent cinquante et un, avril 2003. 
fierai d'exilés-migrants français. Le choix de ces deux parcours est arbitraire et ne vise pas à une quelconque représentativité. C'est surtout le traitement bibliographique et historiographique de ces parcours qui m’intéresse ici. Marie-Antoine Campdoras et Victor Frond quittent la France, l'un pour les Etats-Unis, l'autre pour le Brésil. Le premier ne reviendra pas en France, le second ne séjourne en Amérique du Sud que quelques années. Appartenant au même exil politique, celui des proscrits du Second Empire, la connaissance que nous avons aujourd'hui de leur intégration sociale et politique sur l'autre rive de l'Atlantique diffère non seulement en raison de leur destination et de leur profession mais aussi des différents champs historiographiques qui les ont étudiés. Pour Marie-Antoine Campdoras, c'est sa présence dans les récits qui précédent son départ c'est à dire ceux de la lutte contre le coup d'Etat et le choix de sa destination finale, Topeka, au Kansas, lieu de fondation d'une communauté icarienne où se retrouve un certain nombre de proscrits qui inscrivent son parcours dans l'histoire politique de l'exil ${ }^{11}$. En 1848, il est chirurgien de marine à l'hôpital de Toulon. C'est là qu'il vit les débuts de la Seconde République. Il se mêle cependant peu aux évènements des révolutions de février ou de juin car il embarque sur cinq bateaux différents entre 1848 et 1851 . Il passe ainsi l'essentiel de l'année 1849 (janvier-octobre) sur le navire l'Infernal. Il accomplit ensuite à bord du vaisseau La Provençale, une mission au Sénégal où il débarque le 11 avril 1850. Cette participation aux prémisses de l'aventure coloniale française en Afrique témoigne d'une culture de la mobilité antérieure à son départ vers l'Amérique et parallèle à son engagement politique. Il appartient ainsi à une autre circulation, celle des marins et des militaires qui elle aussi a été longtemps négligée ou du moins dissociée des mouvements migratoires et plus encore politiques ${ }^{12}$. On ignore quel regard il porte sur

${ }^{11}$ Michel Cordillot (dir.), La sociale en Amérique, dictionnaire biographique du mouvement social francophone aux Etats-Unis, Paris, Editions de l'Atelier, 2002 ; René Merle, «Campdoras: de l'espérance républicaine brisée au destin américain», Bulletin de l'Association 1851-2001, n²3, 2003.

${ }^{12}$ Les historiens Léo et Jan Lucassen ont récemment, avec raison, inclus ces professionnels de la mobilité ( migratory labourers) dans l'histoire des migrations. 
la colonisation. C'est donc un homme familier du monde de la mer et qui a déjà quitté à plusieurs reprises l'Europe qui s'exile en 1852. Ces expériences servent certainement son projet mais les sources sont ici muettes. Pourquoi choisit-il l'Amérique ? on l'ignore également. Il s'embarque pour New York, où il arrive le 22 mai tentant l'aventure américaine, dans un monde neuf et démocratique. Campdoras est en effet un républicain convaincu. Dans l'ouvrage, l'Histoire de l'insurrection du Var en décembre 1851, le républicain Noël Blache cite des propos de Campdoras :

"En date du 3 octobre 1852, il[Campdoras] écrit ces lignes :-O triste France, pays de lâches, je ne sais sij'ai pour toi de l'amour ou de la haine !... Hommes de l'ancien, du vieux monde, votre temps est fini; vous êtes en décadence. La France, qui, dit-on, est le cour et le cerveau de l'Europe, est morte. Il n'y a plus rien à espérer d'elle, et sa résurrection est impossible! C'est ici, c'est en Amérique qu'est passée l'âme de l'humanité $^{13}$ »). Ces propos sont politiques, dénonçant la faiblesse de la résistance au coup d'état du futur Napoléon III mais il y ajoute aussi une vision partagée par tous les migrants d'une Amérique comme terre promise.

Son arrivée à New-York ne signifie pas pour autant la fin de son voyage. Il tente de s'y installer en exerçant sa profession de chirurgien pendant plus de deux ans mais sans succès. New-York n'est cependant pas alors une ville si étrangère ou hostile. Des milliers de proscrits et d'émigrés s'y retrouvent. Le jeune chirurgien n'est certainement pas dépaysé dans ce milieu urbain, européen, et fortement politisé. Son départ s'inscrit dans les décennies de forte émigration française vers les Etats-Unis : 130000 entre 1841 et 1850, 110000 entre 1851 et 1860,

13 Noël Blache, Histoire de l'insurrection du Var en décembre 1851, Paris, Le Chevalier, 1869, cité par René Merle, "Campdoras : de l'espérance républicaine brisée au destin américain », Mille-huit cent cinquante et un, Bulletin 23, avril 2003. La réédition de l'ouvrage de Noël Blache qui date de 1983 ne donne pas ce passage. 
72000 entre 1861 et $1870 .{ }^{14}$ Campdoras quitte New York pour la Lousiane où il enseigne pendant quelques mois le français et l'espagnol, il a renoncé à exercer son premier métier. En 1855, il gagne Saint-Louis (Missouri) puis Topeka (Kansas) où il s'installe. On peut penser que ce choix du Kansas implique un désir de rupture avec ces « morceaux d'Europe ", transplantés outre-Atlantique, que sont New York ou la Louisiane. S'agit-il d'une adhésion au mythe américain de la conquête de l'Ouest qui correspondrait à un tempérament d'aventurier ou d'une forme de désengagement politique? Ces deux hypothèses peuvent être retenues sans qu'on en puisse plus particulièrement choisir l'une ou l'autre. Campdoras disparaît alors de l'histoire de l'exil politique. Noel Blache indique en effet dans son livre : «Depuis, nul n'a plus eu de ses nouvelles.... Si jamais ce livre tombe, dans quelque coin du globe, sous les yeux de Campdoras, qu'il n'oublie pas l'appel qu'au nom de ses camarades de jadis, lui adresse l'inconnu qui a signé cet écrit ». Il ne disparaît pas tout à fait car lorsqu'il meurt en 1881, Jules Leroux, frère du socialiste Pierre Leroux, installé en Amérique lui aussi, publie dans son journal californien L'Étoile des pauvres et des souffrants. Organe du communisme libérateur des peuples et de l'Individu son oraison funèbre. Le disparu n’est guère épargné : " Nécrologie. J.B.Campdoras. Je l'ai beaucoup aimé, à distance, en silence. Il tomba dans la mer immonde de la vie du siècle, par imprudence et par excès de zèle ». Cette expression de "mer immonde du siècle" renvoie sans aucun doute au désengagement politique de Campdoras qui n'était plus, aux yeux de l'utopiste Leroux, qu'un migrant plus soucieux des revenus de sa ferme que du communisme libérateur. Pour connaître la réalité de sa vie aux Etats-Unis, ce sont des sources américaines qui nous permettent de connaître sa trajectoire d'exilé. Campdoras devenu farmer n'a pas renoncé pas à toute activité politique et s'engage dans un autre mouvement, américain, celui-ci, le mouvement

14 Olivier Brégeard, «Une communauté fragile, les Français de New York au milieu du XIXème siècle», Annales de démographie historique, $2000 \mathrm{~N}^{\circ} 1$, « Les Français aux Amériques ». p. 43-53. 
des Granges, mouvement d'éducation des fermiers du Sud ${ }^{15}$. En mars 1873, une Convention des fermiers de l'État se réunit à Topeka où les fermiers réclament une réforme du système fiscal, refusent la spéculation, la pression bancaire, l'appropriation du sol par les monopoles ferroviaires. Les Granges du Kansas mettent aussi l'accent sur l'égalité des femmes et des hommes, sur la nécessité d'une éducation sociale et morale. Parmi les neuf délégués de Shawnee County à la Convention, figure le nom de M.A. Campdoras. Dans sa nécrologie, le journal de Topeka est lui élogieux. Il salue « son intense amour de la vérité, de la justice et de la liberté, sa haine de la tyrannie et de l'oppression, sa noblesse de caractère, son dévouement, ses convictions démocratiques et patriotiques ». Il rappelle longuement son engagement de 1851 et cite le récit fait par Noël Blache. En 1882, Eliza Reader, sa veuve, fille de farmer américain, obtient de la République Française une pension de 1000 francs au titre de la loi de réparation nationale de 1881 qui entend indemniser ceux qui ont été victimes de la répression politique de 1851, le chiffre fort élevé. On y apprend qu' il a participé aussi à la guerre de sécession du coté nordiste. Dès l'entrée en guerre, en 1862, Campdoras s' est engagé dans « l’Armée de la Frontière » comme assistant - chirurgien au Second Regiment Kansas Home Guards, « the Indian Regiment » retrouvant son premier métier. Cet engagement n'est pas dénué d'intérêt, l'apport financier régulier de sa solde lui permet aussi, enfin, de faire vivre dignement sa famille. Ici encore comment dissocier engagement et nécessité ? C'est donc hors du champ de l'histoire politique française, par les formes d'intégration socio-professionnelle de Campdoras aux Etats-Unis que l'on peut suivre son adaptation à de nouvelles formes de politisation qui poursuivent ses engagements. Le commentaire de Leroux, qui relève d'une image convenue, celle de l'exilé devenu un migrant qui réussit par l'abandon même de ses idéaux et au prix d'un renoncement à toute volonté de retour doit être infirmé ou pour le moins nuancé. Campdoras a t il un jour songé à revenir en France? Il fait au moins un voyage vers la

${ }^{15}$ René Merle, Mille-huit cent cinquante et un,op cit. 
France en 1880, un an avant sa mort pour se faire soigner en Europe. On ignore là encore si le voyage le ramène vers sa famille et/ou ses anciens compatriotes républicains.

Victor Frond, en 1851, est lui sous-lieutenant à la Caserne de la $4^{\text {e }}$ Compagnie du Bataillon des Sapeurs à Paris. Lors du coup d'état de 1851, il est arrêté et emprisonné en Algérie, il s'échappe et rejoint l'Angleterre. Envoyé en Espagne puis Portugal pour collecter des fonds en vue d'un soulèvement armé en France, il découvre la photographie lors de son séjour à Lisbonne. C'est avec des lettres de recommandation de Victor Hugo qu'il se rend ensuite à Rio de Janeiro où il ouvre un studio de photographie. L'empereur Pierre II lui apporte son soutien : il est le premier photographe de la famille impériale. Il fait venir au Brésil un autre exilé, Ribeyrolles qui a dirigé le journal des proscrits en Grande Bretagne, L'homme. De 1858 à 1860, il réalise avec ce dernier, le Brésil pittoresque ( Brasil pitoresco), ouvrage rédigé en français et en portugais ${ }^{16}$. Après l'amnistie politique de 1859, il revient en France, il poursuit son activité de photographe, chargé de deux collections de 1863 à 1869 : Le Panthéon des illustrations françaises au dix-neuvième siècle et les Actes et histoire du concile cecuménique de Rome. A la naissance de la IIIe république ( le 4 septembre 1870), il est réintégré dans l'armée avec le grade de capitaine du 124 e régiment d'infanterie et reçoit la légion d'honneur le 7 janvier 1871. C'est ici les hasards du militantisme politique qui l'ont conduit hors de France, il se met au service de l'empereur du Brésil ce qui ne semble pas dans l'historiographie française qui connaît mal d'autres histoires nationales, entrer en contradiction avec son républicanisme. Ce que l'on retient c'est à la fois l'innovation dont il fait preuve en matière photographique et la reconnaissance de son action passée par le gouvernement républicain après 1871. Ici les

${ }^{16}$ Lygia Segala, «Prescriptive Observation and Illustration of Brazil : Victor Frond's Photographic Project (1857-61)», Portuguese studies, Volume 23, N 1, 15, March 2007, pp. 55- 70 et Maria Antonia Couto da Silva, «Um espírito imparcial e as paisagens mais belas: considerações acerca da repercussão das imagens do álbum Brasil Pitoresco, de Victor Frond e Charles Ribeyrolles », Revista de historia da arte et arqeologia, $\mathcal{N}^{\circ} 13$, janv-juillet 2010, p 93-106. 
contradictions qui sont plus apparentes que dans le cas de Campdoras sont occultées : Frond est rentré en France, ce qui est une preuve de son rôle d'exilé et non de migrant.

En conclusion, ces deux parcours sont à la fois proches et lointains. Dans un cas, on suit un exilé dont certains républicains déplorent qu'il se fonde dans l'émigration, mais qui prolonge néanmoins clairement son engagement politique d' autres domaines. Dans l'autre, c'est un exilé dont on admire la carrière politique même s'il ne conserve pas plus son engagement politique initial. Qui des deux reste un exilé et qui devient un migrant, qui perd son identité d'exilé ? Ni l'un ni l'autre, ou plutôt les deux. Les deux parcours se complètent. C'est donc à des formes de transferts politiques complexes que l'historien doit accepter d'être confronté. Plutôt que de dissocier les parcours ou au contraire de rechercher les traces d'un lien effectif ou de réseaux pérennes, c'est en montrant comment les répertoires d'action sont mobilisés qu'il convient il me semble de travailler. Il est donc nécessaire de dépasser les questions de quantification ou celle de la réalité des rencontres entre migrants et exilés ou entre exilés et militants des pays d'accueil.

\section{Une communarde et/ou une femme d'affaires en Argentine?}

Les travaux sur le genre et la migration ont permis également d'enrichir notre réflexion sur l'exil ${ }^{17}$. La trajectoire de notre troisième figure française nous en fournit un exemple. Herminie Cadolle est totalement oubliée dans les histoire de la Commune de Paris et de l'exil des communards, comme femme mais aussi parce qu'elle ne cadre pas avec l'histoire malheureuse des exilés. Herminie Cadolle part en Argentine et y fait fortune. C'est par des sources économiques - en travaillant sur des brevets industriels - que j'ai découvert son existence. Herminie, mariée en 1860, est venue s'installer à Paris, avec son mari, et y travaille comme ouvrière corsetière. Durant la Commune de Paris

${ }^{17}$ Donna Gabaccia, From the Other Side. Women, Gender and Immigrant Life in the US, 1820-1990, Bloomington, Indiana University Press, 1994. 
de 1871, elle participe à l'un des premiers mouvement se réclamant du féminisme, l'Union des femmes pour la défense de Paris et les soins des blessés. Elle devient durant les quelques mois de l'insurrection parisienne, l'amie de Louise Michel, une des grandes figures féminines de ce mouvement et de l'anarchisme français. À la fin de l'insurrection, Hermine est arrêtée et emprisonnée à Rouen puis libérée six mois plus tard. Son mari qui est peintre en bâtiment, est condamné à deux ans de prison. Les années suivantes, elle s'implique dans un comité de soutien aux condamnés de la Commune qui ont été déportés en Nouvelle-Calédonie, elle en est la trésorière. Le 10 novembre 1880, elle fait partie des personnalités et amis qui accueille Louise Michel de retour de sa déportation. En 1883, elle vient voir Louise Michel emprisonnée à la suite d'une manifestation. Son fils Alcide est lui-même un militant très actif au sein des mouvements socialistes. Deux personnalités de gauche et anciens communards sont témoins au mariage de son fils, le 28 octobre 1886. Elle décide de quitter la France au début d`année 1887 soit 15 ans après la Commune mais elle fait toujours l'objet de la surveillance policière et est conduite à plusieurs reprises devant les tribunaux. Son départ correspond aussi à son parcours de femme : son mari est mort et son fils marié. Elle s'installe seule en Argentine et ouvre une boutique de lingerie à Buneos Aires. Elle ne se contente pas d'être une bonne commerçante, elle devient aussi une innovatrice. Elle a en effet l'idée de couper le corset en deux pour libérer le corps, de rajouter une armature, et crée ainsi le corselet-gorge, ou maintien-gorge, qui deviendra le soutien gorge. Elle est de retour en France pour présenter ses créations lors de l'exposition universelle de 1889. Elle dépose ensuite un brevet sur le corselet-gorge en 1898 et participe à nouveau à l'exposition universelle de 1900 . En 1910, elle décide de créer un atelier et une boutique à Paris et quitte définitivement l'Argentine. Outre l'invention de l'ancêtre du soutien-gorge, elle est l'une des premières à utiliser une méthode de vente novatrice pour l'époque et observée aux Etats-Unis, la vente par catalogue. Ainsi, au plus fort de son succès au tout début du vingtième siècle, elle fait travailler plus de deux cent ouvrières dans ses ateliers parisiens, ouvrières qui fabriquent de la lingerie aussitôt expédiée à Bueno Aires, Londres, New York ou Saint Pétersbourg... 
C'est une période faste durant laquelle au succès de ses affaires s'ajoutent de nombreuses récompenses à travers le monde. La dénomination d'exilée politique semble dans son cas inappropriée, elle n'est pas condamnée à l'exil et part de France dans les années 1880 soit prés de dix ans après l'échec de la Commune . Elle a malgré tout continué à professer des idées qui restent proches de l'extrême gauche, n'a pas renoncé à ses amitiés passées et est à de nombreuses reprises inquiétée par la police et obligée, avant de choisir de s'embarquer pour l'Amérique du Sud, de comparaitre devant la justice. L'exil est ici différé, séparé de la répression la plus brutale et le départ s'il n'est pas fait dans l'urgence est cependant lié à la surveillance et la suspicion qui ont des répercussions aussi sur sa vie professionnelle. Le choix de Buenos Aires est lié à la fois à l'installation de nombreux exilés dans cette ville mais aussi à l'attrait économique que représente la capitale argentine. Une ville comme Buneos aires est alors un lieu d'arrivée particulièrement civilisé et propice à son activité, la capitale de l'Argentine étant assimilée au Paris de l'Amérique plus que les grandes métropoles d'Amérique du nord. Au delà du caractère presque anecdotique de son histoire, la vie d'Herminie Cadolle témoigne d'une histoire souvent difficile à écrire : celle de femmes qui sont parties et dont on perd souvent la trace car les archives les oublient. La success story est ici exceptionnelle mais elle témoigne de ce que nous ignorons souvent : des liens toujours maintenus avec la France, les allers et retours. Les archives économiques -ici les brevets d'invention- plus que son parcours politique nous offrent de précieuses sources sans lesquelles la trace de la communarde devenue chef d'entreprise serait perdue. Si les conditions de départ précises sont impossibles à connaître dans l'état de nos connaissances, c'est une femme seule et déterminée qui fait le choix de l'Amérique du Sud. Le rapport entre émancipation féminine et mobilité est posé ici à peu près 
dans les mêmes termes dans le cadre des travaux sur les «voyageuses » de Mary Louise Pratt ${ }^{18}$.

\section{Conclusion}

Comme nous avons tenté de le montrer les mobilités choisies et les mobilité subies ne peuvent appartenir à des registres totalement différents. L'expérience vécue de ces mobilités permet de comprendre que les victimes peuvent devenir des acteurs au sens propre, tirant parti de leur déplacement. En proposant des études biographiques variées, il semble possible de dépasser des cloisonnements artificiels. Ceux-ci sont hélas toujours à l'œuvre aujourd'hui lorsque nous parlons des immigrés et des réfugiés. Reste pour approfondir notre connaissance et notre réflexion à confronter aussi des points de vue de part et d'autres de l'Atlantique. Vu d'Europe, il semble encore difficile de penser la question du retour et de considérer ces exilés comme des migrants traversant l'Atlantique dans les deux sens. On néglige aussi beaucoup en Europe, la place des migrants du continent américain et surtout des exilés dans ces mouvements ${ }^{19}$. Leur histoire existe mais notre vision très européo-centrée nous pousse encore à privilégier les routes et les parcours suivis par les Européens et souvent dans un cadre d'étude national voir régional. On se prend à rêver à des rencontres multiples qui ont pu se faire dans les ports et sur les bateaux, entre migrants et exilés en partance et à l'arrivée ${ }^{20}$.

Recebido: 10/08/2017 - Aprovado: 18/09/2017

\footnotetext{
${ }^{18}$ Mary Louise Pratt, Imperial Eyes: Travel Writing and Transculturation, Londres, Routledge, 1992.

19 A l'exception de Delphine Diaz, Jeanne Moisand, Romy Sánchez, Juan Luis Simal (dir.), Exils entre les deux mondes. Migrations et espaces politiques atlantiques au XIX e siècle, Bécherel, Les Perséides, 2015, collection « Le monde atlantique ».

${ }^{20}$ Mémoires du Prince de Talleyrand, Paris, Calmann-Levy, 1891, tome 1, p. 232
} 